\title{
Polyphyletic origins of schizothoracine fish (Cyprinidae, Osteichthyes) and adaptive evolution in their mitochondrial genomes
}

\author{
Takahiro Yonezawa ${ }^{1,2}$, Masami Hasegawa ${ }^{1,2}$ and Yang Zhong ${ }^{1,3 *}$ \\ ${ }^{1}$ School of Life Sciences, Fudan University, SongHu Rd. 2005, Shanghai 200438, China \\ ${ }^{2}$ The Institute of Statistical Mathematics, Midori-cho 10-3, Tachikawa, Tokyo 190-8562, Japan \\ ${ }^{3}$ Institute of Biodiversity Science and Geobiology, Tibet University, JiangSu Rd. 36, Lhasa, 850000, China
}

(Received 25 July 2014, accepted 9 October 2014)

\begin{abstract}
The schizothoracine fish, also called snow trout, are members of the Cyprinidae, and are the most diversified teleost fish in the Qinghai-Tibetan Plateau (QTP). Clarifying the evolutionary history of the schizothoracine fish is therefore important for better understanding the biodiversity of the QTP. Although morphological and molecular phylogenetic studies have supported the monophyly of the Schizothoracinae, a recent molecular phylogenetic study based on the mitochondrial genome questioned the monophyly of this taxon. However, the phylogenetic analysis of that study was on the basis of only three schizothoracine species, and the support values were low. In this report, we inferred the phylogenetic tree on the basis of mitochondrial genome data including 21 schizothoracine species and five closely related species, and the polyphyletic origins of the Schizothoracinae were strongly supported. The tree further suggests that the Schizothoracinae consists of two clades, namely the "morphologically specialized clade" and the "morphologically primitive clade", and that these two clades migrated independently of each other to the QTP and adapted to high altitude. We also detected in their mitochondrial genomes strong signals of positive selection, which probably represent evidence of high-altitude adaptation. In the case of the morphologically specialized clade, positive selection mainly occurred during the Late Paleocene to the Early Oligocene. Its migration also seems to have occurred in the Early Eocene, and this timing is consistent with the drastic uplifting of the QTP. On the other hand, positive selection in the morphologically primitive clade has mainly occurred since the Late Miocene. Because its members are thought to have migrated to the QTP recently, it is possible that they are now undergoing high-altitude adaptation.
\end{abstract}

Key words: phylogeny, snow trout, high-altitude adaptation, Qinghai Tibetan Plateau

\section{MAIN BODY}

Despite being a harsh environment due to its high elevation, the Qinghai Tibetan Plateau (QTP) is known as a hot spot of biodiversity because of its high species richness and abundant endemic species (Myers et al., 2000; Qi et al., 2012). The Schizothoracinae, also called "snow trout", is a subfamily of the Cyprinidae, and comprises 10-13 genera with about 100 species (Mizra, 1991). Schizothoracinae is diagnosed by two lines of enlarged

Edited by Hidenori Nishihara

* Corresponding author. E-mail: yangzhong@fudan.edu.cn Abbreviations: QTP: Qinghai Tibetan Plateau, ML: Maximum Likelihood, Ma: Mega Annum scales along both sides of the anus and urogenital opening (Chen and Chen, 2001). Distributed in the QTP and its surrounding areas, they are the most diversified teleost fish in the QTP, and play important roles in its riverine ecosystems. Therefore, clarifying the evolutionary history of this taxon is important to better understand how the biodiversity of QTP has been configurated.

The schizothoracine fish have been classified into three groups on the basis of morphological characters: the "primitive group", "specialized group" and "highly specialized group" (Cao et al., 1981). The primitive group consists of Schizothorax and Aspiorhynchus. They exhibit more traits similar to the outgroup Barbodes hexagonolepis, and are characterized by having uroneuralia, three or 
four rows of pharyngeal teeth, less degeneration of scales and indistinct special sexual dimorphism. The specialized group consists of Ptychobarbus, Gymnodiptychus and Diptychus, and is characterized by the absence of uroneuralia, one or two rows of pharyngeal teeth, and significant but moderate degeneration of the scales. The highly specialized group consists of Gymnocypris, Oxygymnocypris, Schizophygopsis, Chuanchia and Platypharodon, and is characterized by a total absence of barbels and scales, and a well-developed canalis preoperculomandibularis (Qi et al., 2012). Molecular phylogenetic studies identified two distinct clades in the schizothoracine fish: the first clade comprises the primitive group, and the second clade the specialized and highly specialized groups (He et al., 2004; Qi et al., 2012). Hereafter, we refer to the former as the "morphologically primitive Schizothoracinae clade" and to the latter as the "morphologically specialized Schizothoracinae clade." Although monophyly of the Schizothoracinae has been suggested by morphological and molecular phylogenetic studies (Chen and Chen, 2001; He et al., 2004; Qi et al., 2012), our recent study based on mitochondrial genomes questioned the monophyletic origin of the schizothoracine fish, and suggested that these two groups migrated independently to the QTP in the Early Eocene (the specialized clade) and in the Late Miocene to Pliocene (the primitive clade) ( $\mathrm{Li}$ et al., 2013). However, that study used only three species of schizothoracine fish, and failed to establish robust phylogenetic relationships. Since Qi et al. (2012) reported many examples of parallel evolution (common ancestral character states in different lineages evolve independently into other common character states) and convergent evolution (different ancestral character states in different lineages evolve into common character states) in the morphological characters of the Schizothoracinae, it is necessary to reexamine the taxonomic status of this subfamily with a more abundant taxon sampling.

In this study, we reconstructed the phylogenetic tree on the basis of mitochondrial genome data including 21 species of schizothoracine fish and five closely related species. Detailed methods for phylogenetic inference and other analyses are provided in the Supplementary MATERIALS AND METHODS. The maximum likelihood (ML) tree is shown in Fig. 1. The morphologically specialized schizothoracine clade and the Barbinae sensu stricto (Barbus barbus and Luciobarbus capito) were

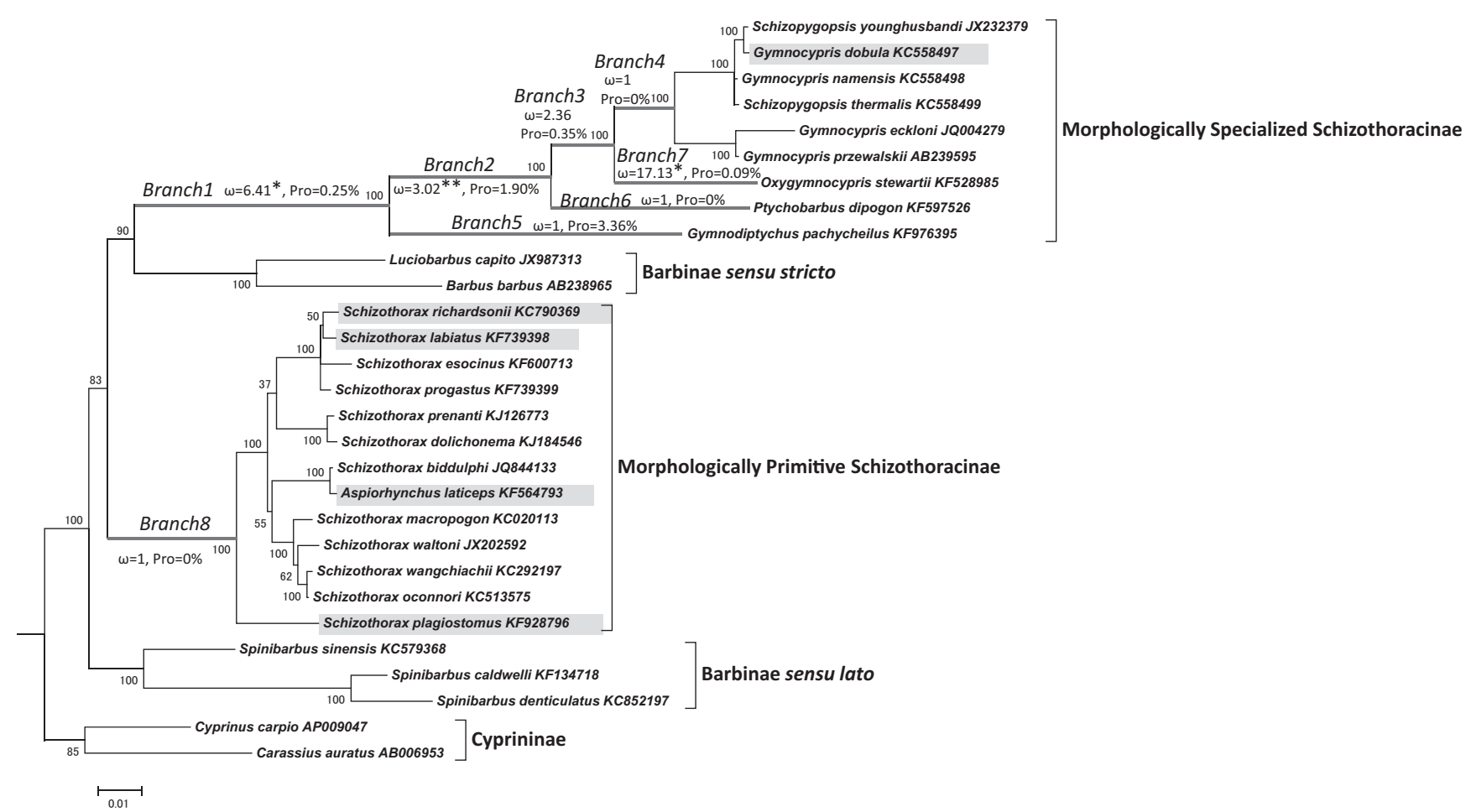

Fig. 1. The ML tree as inferred from mitochondrial genomes is shown. In this figure, only Schizothoracinae and its closely related species are shown. The whole picture of the ML tree is indicated in Supplementary Fig. S1. The branch lengths are proportional to the numbers of amino acid (for 12 protein-coding genes) and nucleotide (12S rRNA and 16S rRNA) substitutions. The scale bar shows the number of substitutions per site. Nodal numbers indicate bootstrap probabilities (rapid bootstrap algorithm: 100 replications). Branches indicated by bold gray lines are deep (> about $10 \mathrm{Ma}$ ) ancestral branches in which the branch site model was applied. $\omega$ values and the proportions of positively selected sites (Pro) are indicated along the respective branches. One (*) and two asterisks $(* *)$ indicate $\omega$ values higher than 1 at significance levels of $5 \%$ and $1 \%$, respectively. Species with shaded names were under positive selection in relatively recent times (< about $10 \mathrm{Ma})$. 
grouped into monophyly (90\%), and the morphologically primitive schizothoracine clade was placed as their sister group. The bootstrap probability supporting monophyly of the Schizothoracinae was only $3.5 \%$, and the monophyly hypothesis was marginally rejected (the P-value of the Kishino-Hasegawa test is 4.6-5.3\%) (Table 1). The genus Spinibarbus (the Barbinae sensu lato) was recognized as a monophyletic group, and was placed as a sister group of the schizothoracine fish + Barbinae sensu stricto. This is contrary to $\mathrm{Li}$ et al. (2013)'s cytochrome b-based tree that indicated Spinibarbus is a polyphyletic group, and Spinibarbus (Spinibarbichthys) sinensis is closer to the primitive schizothoracine clade than to other Spinibarbus species. Cyprininae (Cyprinus and Carassius) was placed as the most basal lineage among Schizothoracinae + Barbinae sensu stricto + Spinibarbus.

This phylogenetic hypothesis is basically consistent with our previous study ( $\mathrm{Li}$ et al., 2013, except for Spinibarbus) with higher nodal supports. The subfamily Schizothoracinae is polyphyletic, and the taxonomic validity of this group should be reexamined. Li et al. (2013) reconstructed the geographical distribution areas of the ancestral species, and concluded that the morphologically specialized clade and the primitive clade of the schizothoracine fish seemed to have migrated independently to the QTP. The morphologically specialized clade originated in West Asia-Europe (probably the coast of Tethys Ocean) and the primitive clade originated in East and Southeast Asia. Since ancestral state reconstructions are highly dependent on tree topologies, the main issue of their evolutionary hypothesis was the weak support values of their tree topology.

Therefore, the robust phylogenetic tree inferred by this study confirmed Li et al. (2013)'s evolutionary scenario that the two groups independently migrated into the QTP and have experienced parallel evolution with respect to high-altitude adaptation. The many parallel and convergent morphological changes in the schizothoracine fish (Qi et al., 2012) also support this scenario.

The harsh environment of the QTP is characterized by cold and hypoxic conditions. Since the mitochondria play important roles in synthesizing ATP and generating heat by aerobic respiration, it is plausible that the mitochondrial genomes of the schizothoracine fish have experienced adaptive evolution. Interestingly, a weak signal of positive selection was detected in the ancestral branch

Table 1. Comparison of tree topologies among four taxa

\begin{tabular}{|c|c|c|c|c|c|c|}
\hline Tree & Topologies $^{1}$ & $\operatorname{lnL^{2}}$ & $\mathrm{KH}^{3}$ & $\overline{\mathrm{AU}^{4}}$ & $\mathrm{PP}^{5}$ & $\mathrm{BP}^{6}$ \\
\hline 1 & (((SS,Bar),PS),Spi) & $<-43030.481>$ & - & 0.957 & 1 & 0.799 \\
\hline 2 & ((SS,Bar),(PS,Spi)) & -7.7 & 0.124 & 0.202 & $4.00 \mathrm{E}-04$ & 0.105 \\
\hline 3 & (((SS,Bar),Spi),PS) & -9.9 & 0.055 & 0.03 & $5.00 \mathrm{E}-05$ & 0.006 \\
\hline 4 & (((SS,PS),Bar),Spi) & -18.1 & 0.046 & 0.072 & $1.00 \mathrm{E}-08$ & 0.011 \\
\hline 5 & (((PS,Bar),SS),Spi) & -18.8 & 0.041 & 0.048 & $7.00 \mathrm{E}-09$ & 0.01 \\
\hline 6 & ((SS,PS),(Bar,Spi)) & -21.0 & 0.053 & 0.112 & $8.00 \mathrm{E}-10$ & 0.024 \\
\hline 7 & (((SS,PS),Spi),Bar) & -21.5 & 0.049 & 0.097 & $5.00 \mathrm{E}-10$ & 0.014 \\
\hline 8 & $(((\mathrm{Bar}, \mathrm{Spi}), \mathrm{PS}), \mathrm{SS})$ & -23.4 & 0.051 & 0.099 & $7.00 \mathrm{E}-11$ & 0.02 \\
\hline 9 & $(((\mathrm{PS}, \mathrm{Bar}), \mathrm{Spi}), \mathrm{SS})$ & -23.4 & 0.036 & 0.076 & $7.00 \mathrm{E}-11$ & 0.009 \\
\hline 10 & (((Bar,Spi),SS),PS) & -26.3 & 0.022 & 0.015 & $4.00 \mathrm{E}-12$ & $3.00 \mathrm{E}-04$ \\
\hline 11 & (((PS,Spi),Bar),SS) & -26.4 & 0.026 & 0.024 & $3.00 \mathrm{E}-12$ & 0.001 \\
\hline 12 & $((\mathrm{SS}, \mathrm{Spi}),(\mathrm{PS}, \mathrm{Bar}))$ & -26.4 & 0.017 & 0.012 & $3.00 \mathrm{E}-12$ & $3.00 \mathrm{E}-04$ \\
\hline 13 & (((PS,Spi),SS),Bar) & -29.2 & 0.015 & 0.003 & $2.00 \mathrm{E}-13$ & $6.00 \mathrm{E}-05$ \\
\hline 14 & (((SS,Spi),PS),Bar) & -29.2 & 0.015 & 0.005 & $2.00 \mathrm{E}-13$ & 0 \\
\hline 15 & (((SS,Spi),Bar),PS) & -31.1 & 0.005 & 0.002 & $3.00 \mathrm{E}-14$ & 0 \\
\hline
\end{tabular}

Schizothoracinae monophyletic trees are shaded

${ }^{1}$ Topologies:

SS: morphologically Specialized Schizothoracinae

PS: morphologically Primitive Schizothoracinae

Bar: Barbinae sensu stricto

Spi: Spinibarbus

${ }^{2} \operatorname{lnL}$ : Total log likelihood score of the 12 protein-coding genes and 2 ribosomal RNA genes

The absolute value of $\operatorname{lnL}$ is shown only for the ML tree in parentheses <>. The differences of the lnLs compared to that of the ML tree are indicated for the alternative trees

${ }^{3} \mathbf{K H}$ : P-values of the Kishino-Hasegawa test

${ }^{4} \mathbf{A U}$ : P-values of the Approximate Unbiased test

${ }^{5}$ PP: Bayesian Posterior Probabilities of the tree topologies

${ }^{6} \mathbf{B P}$ : Bootstrap probabilities of the tree topologies calculated by the RELL method 
of the morphologically specialized clade (Li et al., 2013); this ancestral branch corresponds to branch1-branch4 in Fig. 1. However, Li et al. (2013)'s taxon sampling was too sparse, and failed to obtain a significant result ( $\mathrm{P}$ value of the likelihood ratio test (LRT) was $8 \%$ ). In this study, several species of distinct lineages such as Gymnodiptychus, Ptychobarbus and Oxygymnocypris were included within the morphologically specialized Schizothoracinae, and these species can cut the long ancestral branch into several short branches (branch1branch4). We applied the branch site model, which allows heterogeneity of the $\omega(\mathrm{dN} / \mathrm{dS})$ ratio among the sites along specific branches (Yang and Nielsen, 2002). Positive selection was detected in branch1, branch2, branch3 and branch7, its signals in branch1, branch2 and branch7 being statistically significant ( $\mathrm{P}$ values of the LRT are $2.26 \%$ for branch1, 3.46E-6\% for branch2 and $4.8 \%$ for branch7; Table 2). Positively selected sites are summarized in Supplementary Table S1. cytochrome b, NADH2, NADH4 and NADH5 were under positive selection in branch1. ATP6, NADH2 and NADH5 were under positive selection in branch2. ATP6 and cytochrome $b$ were under positive selection in branch3. NADH2, NADH3 and NADH5 were under positive selection in branch7. Positive selection seemed mainly to be operating on respiratory chain complexes I (NADH1-6) and III (cytochrome b). It is known that mitochondrial reactive oxygen species (ROS) are mainly generated at complex I and III, and hypoxia particularly increases ROS generation at complex III (Murphy, 2009). Therefore, it is possible that positive selection on the mitochondrial genomes of these fish reflects adaptive evolution to the hypoxic environment at high altitudes. Interestingly, Guan et al. (2014) also reported positive selection of hypoxia-inducible factor alpha B (HIF- $\alpha \mathrm{B})$, which is an important regulator of gene expression under hypoxic conditions, in the morphologically specialized Schizothoracinae clade.

Branch 1 is the common ancestor of the morphologically specialized clade. It corresponds to 58.0-42.5 Mega Annum (Ma; million years ago) (Li et al., 2013), and this clade seems to have migrated to the QTP during this time. More accurately, the timing of the migration should be after the branching out of Cyprinion, which is currently distributed in West Asia and South Asia, at $53.0 \mathrm{Ma}$ (Li et al., 2013). It is thought that the collision of the Indian subcontinent and Eurasian continent, and the first major uplifting of the QTP, occurred in the Paleocene to the Early Eocene, 58-53 Ma (Zhang et al., 2013). The schizothoracine migration to the QTP (53.042.5 Ma) seems to have occurred after these geological events. Branch2 is the common ancestral branch of Ptychobarbus, Oxygymnocypris, Gymnocypris and Schizopygopsis, and corresponds to 42.5-32.0 $\mathrm{Ma}$ ( $\mathrm{Li}$ et al., 2013). This timing is consistent with the second
Table 2. Results of the branch site model

\begin{tabular}{|c|c|c|c|c|c|}
\hline & & $\begin{array}{c}\text { site } \\
{\text { class } 1^{1}}^{2}\end{array}$ & $\begin{array}{c}\text { site } \\
\text { class2 }^{2}\end{array}$ & 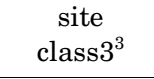 & $\begin{array}{c}\text { site } \\
\text { class }^{4}\end{array}$ \\
\hline & foreground $^{5}$ & 0.02736 & 1 & $6.41167^{*}$ & $6.41167^{*}$ \\
\hline \multirow[t]{3}{*}{ branch1 } & background ${ }^{6}$ & 0.02736 & 1 & 0.02736 & 1 \\
\hline & proportions $^{7}$ & 0.95288 & 0.04464 & 0.00237 & 0.00011 \\
\hline & foreground $^{5}$ & 0.02709 & 1 & $3.02162^{* *}$ & $3.02162 * *$ \\
\hline \multirow[t]{3}{*}{ branch2 } & background ${ }^{6}$ & 0.02709 & 1 & 0.02709 & 1 \\
\hline & proportions $^{7}$ & 0.93799 & 0.04304 & 0.01814 & 0.00083 \\
\hline & foreground $^{5}$ & 0.02739 & 1 & 2.3613 & 2.3613 \\
\hline \multirow[t]{3}{*}{ branch3 } & background $^{6}$ & 0.02739 & 1 & 0.02739 & 1 \\
\hline & proportions ${ }^{7}$ & 0.95181 & 0.04474 & 0.0033 & 0.00015 \\
\hline & foreground $^{5}$ & 0.02745 & 1 & 1 & 1 \\
\hline \multirow[t]{3}{*}{ branch4 } & background ${ }^{6}$ & 0.02745 & 1 & 0.02745 & 1 \\
\hline & proportions $^{7}$ & 0.95507 & 0.04493 & 0 & 0 \\
\hline & foreground $^{5}$ & 0.02678 & 1 & 1 & 1 \\
\hline \multirow[t]{3}{*}{ branch5 } & background ${ }^{6}$ & 0.02678 & 1 & 0.02678 & 1 \\
\hline & proportions $^{7}$ & 0.92308 & 0.04332 & 0.0321 & 0.00151 \\
\hline & foreground $^{5}$ & 0.02770 & 1 & 1 & 1 \\
\hline \multirow[t]{3}{*}{ branch 6} & background ${ }^{6}$ & 0.02770 & 1 & 0.02770 & 1 \\
\hline & proportions $^{7}$ & 0.95865 & 0.04135 & 0 & 0 \\
\hline & foreground $^{5}$ & 0.02768 & 1 & $17.12744^{*}$ & $17.12744^{*}$ \\
\hline \multirow[t]{3}{*}{ branch 7} & background ${ }^{6}$ & 0.02768 & 1 & 0.02768 & 1 \\
\hline & proportions ${ }^{7}$ & 0.95861 & 0.04050 & 0.00086 & 0.00004 \\
\hline & foreground $^{5}$ & 0.02745 & 1 & 1 & 1 \\
\hline \multirow[t]{2}{*}{ branch 8} & background $^{6}$ & 0.02745 & 1 & 0.02745 & 1 \\
\hline & proportions $^{7}$ & 0.95508 & 0.04492 & 0 & 0 \\
\hline
\end{tabular}

The branch site model allows heterogeneity of the $\omega(\mathrm{dN} / \mathrm{dS})$ ratio among sites along specific branches. This model assumes that there are two kinds of branches, namely foreground branches and background branches. There are purifying selection sites, neutral selection sites, and positive selection sites in the foreground, and there are only purifying selection sites and neutral selection sites in the background (no positive selection sites).

'site class1: In this site class, both the foreground and the background are under purifying selection.

site class2: In this site class, both the foreground and the background are under neutral selection.

${ }^{3}$ site class3: In this site class, the foreground is under positive selection, and the background is under purifying selection.

${ }^{4}$ site class4: In this site class, the foreground is under positive selection, and the background is under neutral selection.

${ }^{5}$ foreground: The $\omega$ ratios of the foreground.

${ }^{6}$ background: The $\omega$ ratios of the background.

${ }^{7}$ proportions: The ratios of the corresponding amino acid sites in each site class among all sites.

Asterisks indicate statistical significance.

* The P-value of the LRT $<0.05$.

** The P-value of the LRT $<0.01$.

major uplifting of the QTP (45-30 Ma: Zhang et al., 2013). Rowley and Currie (2006a, 2006b) suggested the QTP has been at an elevation of more than $4000 \mathrm{~m}$ since $35 \mathrm{Ma}$ (but see also Kitoh, 2005; Molnar et al., 2006). 
The drastic convulsion of the environment may have triggered the adaptive evolution of this clade. The positive selection operating in branch2 was much stronger than that in branch1: whereas only $0.25 \%$ of the amino acid sites in the mitochondrial genome were under positive selection in branch1, $1.90 \%$ were under positive selection in branch2. Major high-altitude adaptation of the morphologically specialized schizothoracine fish seems to have occurred during this second uplift period. The genera Gymnodiptychus and Diptychus, which are the basal group of the specialized clade, are distributed not only in the QTP but also widely in China. We also failed to detect a positive selection signal in the branch of Gymnodiptychus (branch5). The divergence time of Gymnodiptychus and other morphologically specialized schizothoracine fish was estimated to be $42.5 \mathrm{Ma}$ (the Early Eocene) by Li et al. (2013). This suggests that the common ancestor of the morphologically specialized schizothoracine fish migrated to the QTP in the Early Eocene, adapted weakly to the high altitude, and then adapted more strongly to the higher altitude with the drastic uplifting of the QTP in the Late Eocene. On the other hand, intensive Qinghai-Tibetan movement in the Neogene has also been suggested (Fang et al., 1997). The positive selection detected in Oxygymnocypris (branch7) and G. dobula (see Supplementary RESULTS AND DISCUSSION) is suggestive of adaptation during this period.

In contrast, we failed to detect positive selection of mitochondrial genomes in the ancestral branch of the morphologically primitive clade (branch8; Table 2). Interestingly, Guan et al. (2014) also detected positive selection of HIF- $\alpha \mathrm{B}$ in the morphologically specialized clade, but not in the morphologically primitive clade. This is probably due to the difference in altitude distribution areas: the morphologically specialized clade is distributed in higher regions $(2500-4500 \mathrm{~m})$ than the primitive clade (1500-2500 m) (Guan et al., 2014). In addition, the primitive clade appears to have migrated to the QTP relatively recently (later than the Middle Miocene, $10 \mathrm{Ma}$; Li et al., 2013). Therefore, the degree of high-altitude adaptation of the primitive clade seems still to be low.

However, we detected positive selection signals in the terminal branches of $S$. plagiostomus, S. labiatus, $S$. richardsonii, A. laticeps (Fig. 1; see Supplementary RESULTS AND DISCUSSION). It is therefore likely that these species are now undergoing high-altitude adaptation. The example of the Schizothoracinae reveals correlations between geological events in the QTP and the major evolutionary events experienced by the organisms distributed in this area.

This work was supported by NSFC 30925004, NSFC 91131901, and the PCSIRT project to Y.Z., NSFC ISIS584763SN:450050 and the Transdisciplinary Research Integration Center (TRIC) of the Research Organization of Information and Systems to T.Y., and by JSPS Grants-in-Aid for Scientific Research C25440219 to M.H.

\section{REFERENCES}

Cao, W., Chen, Y., Wu, Y., and Zhu, S. (1981) Origin and evolution of schizothoracine fishes in relation to the upheaval of the Xizang Plateau. In: Studies on the Period, Amplitude and Type of the Uplift of the Qinghai-Xizang Plateau (ed: Tibetan Expedition Team of the Chinese Academy of Science), pp. 118-130. Science Press, Beijing (in Chinese).

Chen, Z. M., and Chen, Y. F. (2001) Phylogeny of the specialized Schizothoracine fishes (Teleostei: Cypriniformes: Cyprinidae). Zoological Studies 40, 147-157.

Fang, X. M., Li, J. J., Zhu, J. J., Chen, H. L., and Cao, J. X. (1997) The absolute age and division of Cenozoic stratum from the Linxia Basin in Gansu Province. Chin. Sci. Bull. 42, 1458-1471 (in Chinese).

Guan, L., Chi, W., Xiao, W., Chen, L., and He, S. (2014) Analysis of hypoxia-inducible factor alpha polyploidization reveals adaptation to Tibetan plateau in the evolution of schizothoracine fish. BMC Evolutionary Biology 14, 192.

He, D., Chen, Y., and Chen, Y. (2004) Molecular phylogeny of the specialized schizothoracine fishes (Teleostei: Cyprinidae), with their implications for the uplift of the Qinghai-Tibetan Plateau. Chin. Sci. Bull. 49, 39-48.

Kitoh, A. (2005) Climate model simulation on the role of mountain uplift on Asian monsoon. J. Geol. Soc. Japan 111, 654-667 (in Japanese).

Li, Y., Ren, Z., Shedlock, A. M., Wu, J., Sang, L., Tersing, T., Hasegawa, M., Yonezawa, T., and Zhong, Y. (2013) High altitude adaptation of the schizothoracine fishes (Cyprinidae) revealed by the mitochondrial genome analyses. Gene $\mathbf{5 1 7}$, 169-178.

Mirza, M. R. (1991) A contribution to the systematics of the Schizothoracine fishes (Pisces: Cyprinidae) with the description of three new tribes. Pakistan J. Zool. 23, 339-341.

Molnar, P., Houseman, G. A., and England, P. C. (2006) Palaeoaltimetry of Tibet. Nature 444, doi: 10.1038/nature05368.

Murphy, M. (2009) How mitochondria produce reactive oxygen species. Biochem. J. 417, 1-13.

Myers, N., Mittermeier, R. A., Mittermeier, C. G., da Fonseca, G. A. B., and Kent, J. (2000) Biodiversity hotspots for conservation priorities. Nature 403, 853-858.

Qi, D., Chao, Y., Guo, S., Zhao, L., Li, T., Wei, F., and Zhao, X. (2012) Convergent parallel and correlated evolution of trophic morphologies in the subfamily Schizothoracinae from the Qinghai-Tibetan Plateau. PLoS One 7, e34070.

Rowley, D. B., and Currie, B. S. (2006a) Palaeo-altimetry of the late Eocene to Miocene Lunpola basin, central Tibet. Nature 439, 677-681.

Rowley, D. B., and Currie, B. S. (2006b) Palaeo-altimetry of Tibet (reply). Nature 444, doi:10.1038/nature05369.

Yang, Z., and Nielsen, R., (2002) Codon-substitution models for detecting molecular adaptation at individual sites along specific lineages. Mol. Biol. Evol. 19, 908-917.

Zhang, K., Wang, G., Yu, Y., Luo, M., Ji, J., Xiao, G., Wang, A., Song, B., Liang, Y., Jiang, S., et al. (2013) Sedimentary evolution of the Qinghai-Tibet Plateau in Cenozoic and its response to the uplift of the plateau. Acta Geol. Sin. Engl. 87, $555-575$. 